\title{
Unsteady shrinking sheet with mass transfer in a rotating fluid
}

\begin{abstract}
In this paper, the problem of unsteady flow induced by a shrinking sheet with mass transfer in a rotating fluid is studied. The transformed boundary layer equations are solved numerically by an implicit finite-difference scheme known as the Keller-box method. The influence of rotation, unsteadiness and mass suction parameters on the reduced skin friction coefficients $\mathrm{f}$ njo) and $g\left(\frac{D}{)}\right)$, as well as the lateral velocity and velocity profiles are presented and discussed in detail.
\end{abstract}

Keyword: Unsteady pow; Boundary layer; Mass transfer; Rotating puid; Shrinking sheet; Numerical solution 